\title{
The Remote Control System of Domestic Robots Based on Android
}

\author{
Xinhua Lin, Yannan Jiang and Yulian Jiang* \\ College of Electrical and information Engineering, Southwest MinZu University, Chengdu, Sichuan, 610225, P.R. China \\ ${ }^{*}$ Corresponding Author
}

\begin{abstract}
Based on self-developed and somatosensorymapping robot, a remote control system of domestic robots is designed in this paper, in which the system adopts intelligent speech recognition technology in terms of $\mathrm{C} / \mathrm{S}$ framework such that the speech control robot has been used to do some basic housework. Combining with HTTP and MQTT, the robot can be employed to do the video surveillance and environmental monitoring. By delivering video stream and environmental parameters to Android devices, users can remotely monitor house situation easily, which makes the control of domestic robots smarter and easier.
\end{abstract}

Keywords-domestic robots; android; remote control; Speech recognition

\section{INTRODUCTION}

With the aggravating trend of aging population, more elderly people need to be card. However, due to fast-paced lifestyles and heavy work pressure, children do not have enough time to spend with their parents, which makes the need of social security more urgent. Therefore, a huge demand for domestic robots generates.

With the development of robotics and Internet technology, domestic robots have been accepted by more families. The remote control of robots is a hot issue in the field of current robot research, which not only makes robot-operating more convenient, but also plays an important role in promoting smart application of robots. In this paper, the use of remote control system based on Android allows the operator to remotely control the movement of robots and grab items easily. Meanwhile, robotic sensors and camera can be used to remotely monitor house situation and air indicators in real time. When a series of dangers such as fire occur, the robot can alert the user in time.

\section{ROBOT HARDWARE STRUCTURE}

In this paper, the robot is self-developed by our project team and uses the somatosensory-mapping technology [1-3]. The operator does an action by wearing data gloves with bend sensors, gyroscope and STM32. We use the Kalman filter algorithm to calculate the data collected by sensors, and obtain the data of movement angle and position of arms. These data can be transported to robotic master microcontroller through the sever, and the microcontroller generates the PWM to control the movement of each servo in robotic manipulators, which achieves a synchronized movement between manipulators and human hands. The robot is shown in Figure I. To meet the requirements of crawling different shape items, the left hand of robot is a bionic mechanical claw and the right hand is a mechanical clip. Robotic walking system is wheeled structure. In order to make it more convenient to operate the robot, in this paper, we design a remote control system based on Android.

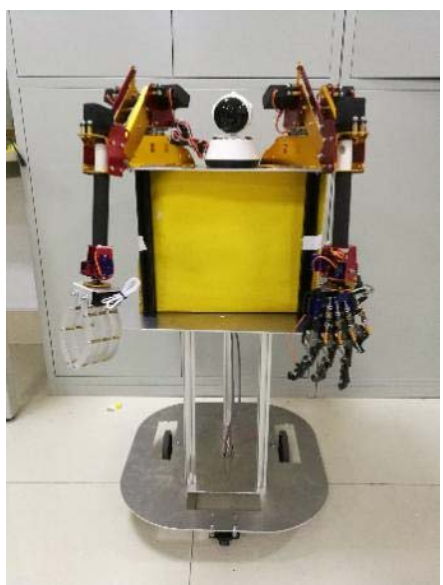

FIGURE I. ROBOT HARDWARE STRUCTURE

\section{The DESIGN OF ANDROID CONTROL SySTEM}

\section{A. The Overall Structure of the System}

The whole system adopts C / S framework, that is, the user initiates a request to the server, and the server accepts the request, and then returns the data to the user. The system consists of intelligent speech control system and environmental monitoring system, where the environmental monitoring system consists of environmental parameter detection system and video surveillance system shown in Figure II.

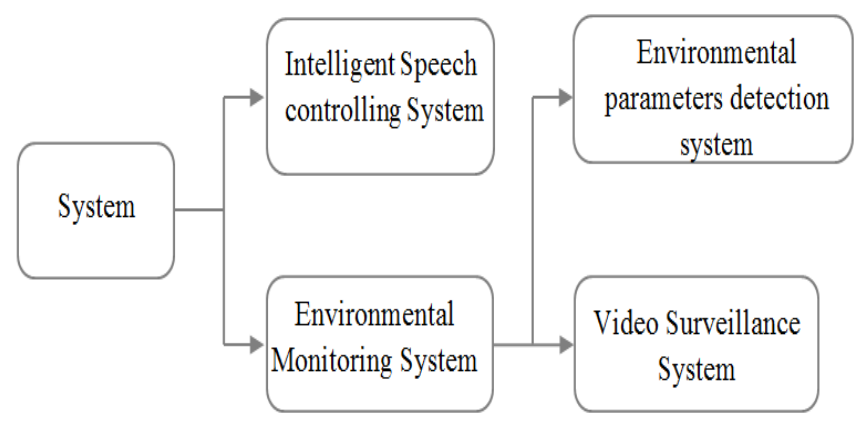

FIGURE II. THE STRUCTURE OF THE SYSTEM 
The camera that robot is equipped with can be used for capturing the real-time picture of home. Meanwhile, the robot can collect environmental parameters in real time by equipping with temperature sensor, humidity sensor and smoke sensor. Through the wireless network transmission, the above video stream and environmental parameters can be displayed on the robot remote control interface. The remote control process is shown in Figure III.

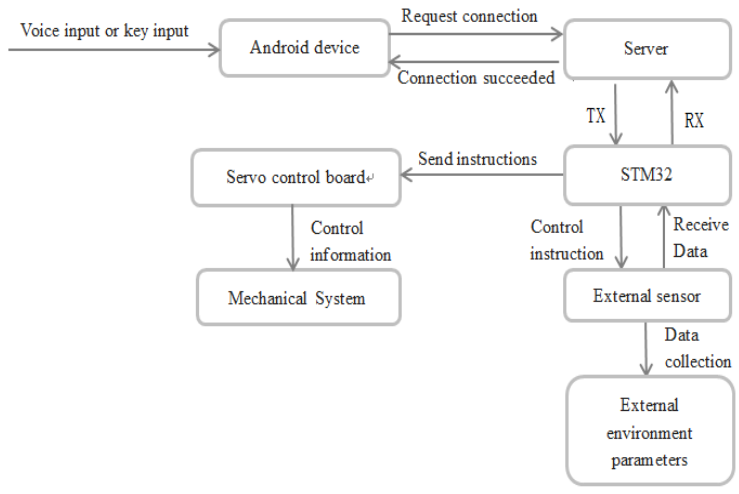

FIGURE III. REMOTE CONTROLFLOW CHART

\section{B. Intelligent Speech Control System}

In this paper, the intelligent speech control system [4-6] adopts the speech recognition technology of IFLYTEK [7-9], and combines with Struts2 framework, OKHTTP Network request framework and Litepal database framework realizing the intelligent recognition of the user's speech. The intelligent speech recognition interface is shown in Figure IV.

Through speech recognition technology, user's speech codes can be accurately converted into words. Struts2 framework can put the project into web server and operate the data base. By using the callback technology of OKHTTP, we can get the data processing program from server to client local system. After that, the LitePal database framework can be used to operate the local database so that the recognized words can be updated to the server, which not only enables extranet access to the database, but also makes program code more succinct. The process is shown in Figure V.

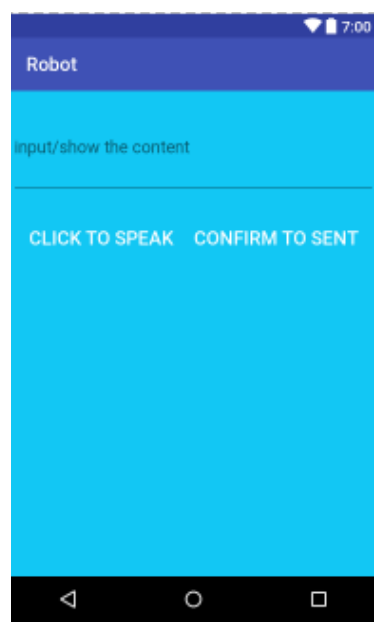

FIGURE IV. VOICE INPUT OF CLIENT

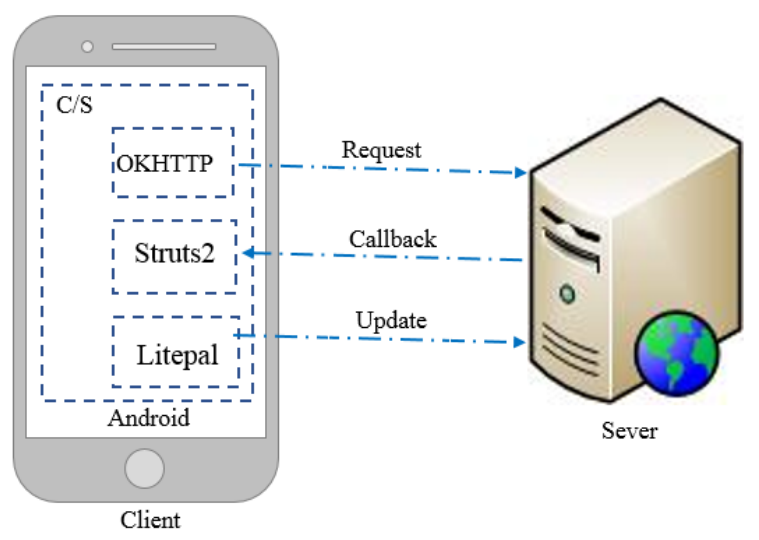

FIGURE V. SPEECH RECOGNITION SYSTEM WORK DIAGRAM

In this paper, speech is recognized and converted into words, then the words are sent to the database. If the words match the existing words in the database, the server will send instructions to the robot's STM32 master chip, and then STM32 controls the robot to perform certain actions, which realizes the intelligent speech control robot. The flow chart of speech recognition is shown in Figure VI.

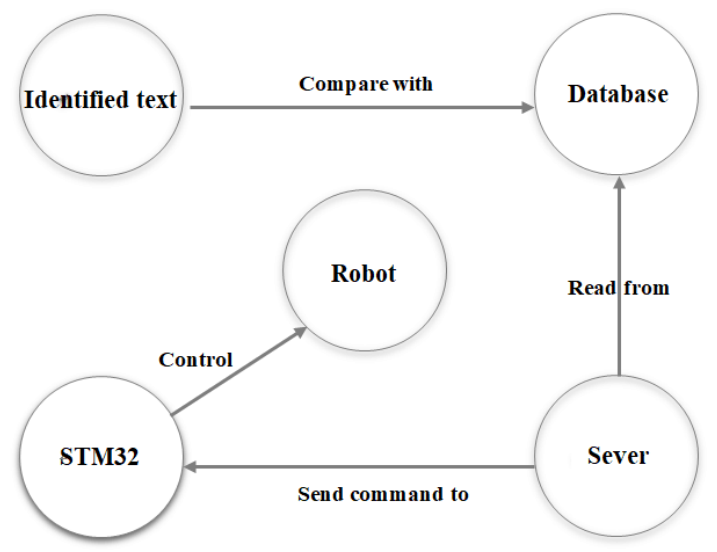

FIGURE VI. THE FLOW CHART OF SPEECH RECOGNITION

\section{ENVIRONMENTAL MONITORING SYSTEM}

\section{A. Environmental Parameters Detection System}

The temperature sensor, humidity sensor [10,11] and smoke sensor installed in the robot can collect the environmental parameters and send data to the server by STM32 and MQTT protocol [12,13]. In order to make users more convenient to observe the environmental parameters, in the process of receiving data, the same way of receiving data is used. When the environmental parameters are abnormal, that is, a series of dangers such as fire occur, the robot can alert the user in time.

The reason why we choose MQTT as data transmission protocol is that we do not need to deal with the environmental parameters, so we do not need to program in server, which increases the efficiency of data transmission and reduces the transmission time. It can provide real-time, reliable messaging services to connect remote devices with a little code and 
limited bandwidth. The implementation process is shown in Figure VII.

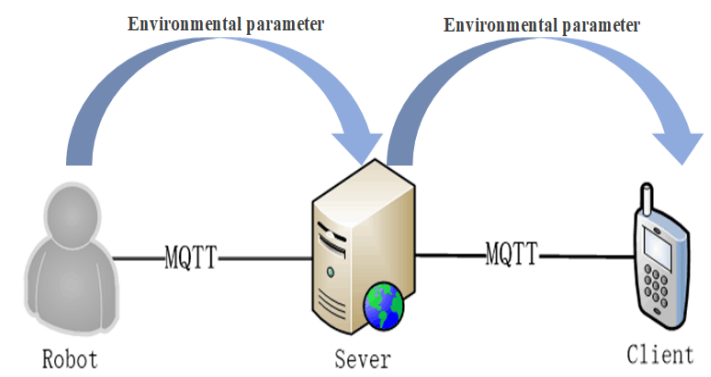

FIGURE VII. IMPLEMENTATION OF ENVIRONMENTAL PARAMETER DETECTION SYSTEM

\section{B. Video Surveillance System}

Video surveillance system $[14,15]$ can transport the home video stream to Android devices, which makes users monitor home situation in real time. In this paper, the robot is installed with the webcam based on HTTP and TCP, which can encapsulate video stream data into HTTP data groups and provide the web access function, and then the client uses the TCP to get the video stream. The implementation process is shown in Figure VIII.

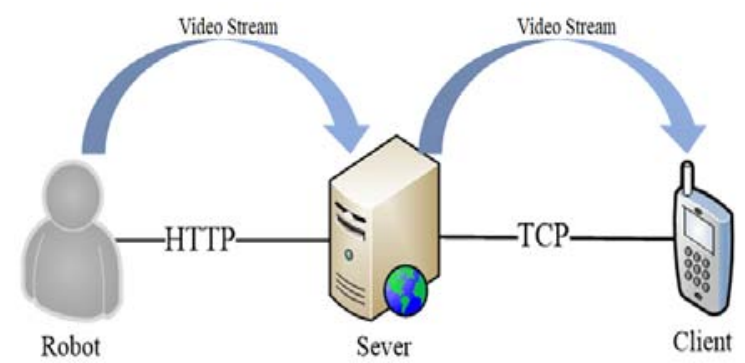

FIGURE VIII. IMPLEMENTATION OF VIDEO SURVEILLANCE SYSTEM

The client interfaces of the environmental monitoring system include the interface of environment parameters monitoring and video surveillance, which are shown in Figures IX and X, respectively.

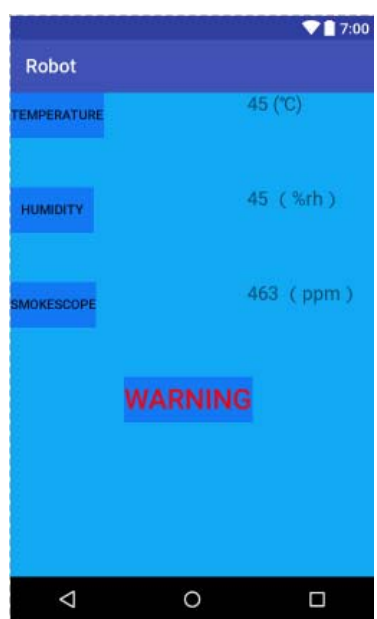

FIGURE IX. THE INTERFACE OF ENVIRONMENT

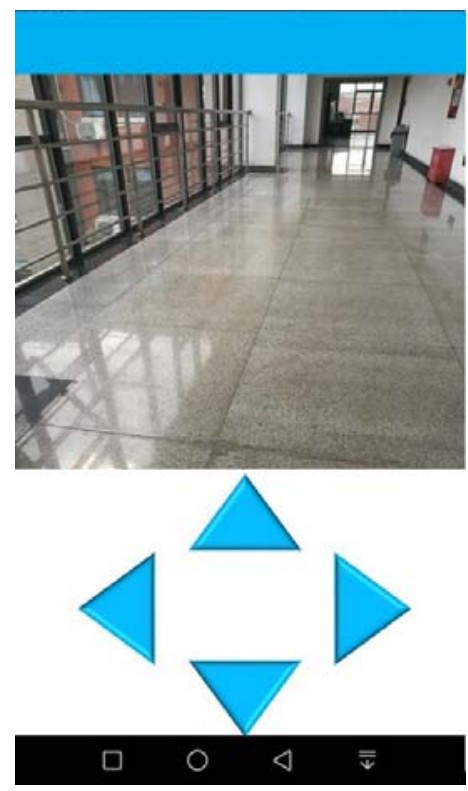

FIGURE X. THE INTERFACE OF VIDEO SURVEILLANCE PARAMETERS MONITORING

Figure 9 shows the values of temperature, humidity and smoke concentration, and when the value exceeds the preset normal value, the warning prompt as shown in Figure IX will appear. Figure $\mathrm{X}$ shows the monitoring picture and the keys which control the robot to walk.

\section{CONCLUSION}

In this paper, we build the robot remote control system based on Android in terms of $\mathrm{C} / \mathrm{S}$ framework. The control system is composed of intelligent speech control system and environmental monitoring system. The intelligent speech control system can accurately recognize the natural speech of users and can convert speech into words, and then compared the recognized words with the preset words in the database, if they match together, the server will send corresponding instructions to control robotic movement. The environmental monitoring system includes environmental parameters detection system and video surveillance system. The environmental parameters detection system can send the values of temperature, humidity and smoke concentration that robot probes at the moment to the client. When the value exceeds the preset normal value, the robot can alert the user, which makes users deal with dangerous situation in time. The video surveillance system can transport home monitoring picture to users in real time and there is no delay in the process of video transmission. After several experiments, it can be proved that the remote control system in this paper can achieve above mentioned functions, which enables users to grasp the home situation quickly and conveniently.

\section{ACKNOWLEDGMENT}

This research was supported by the Fundamental Research Funds for the Central Universities, Southwest Minzu University (NO.2016NZYQN07). 


\section{REFERENCES}

[1] Zhang Wei-jie. Structural Design and Characterization of a New Exoskeleton Finger Rehabilitation Robot[D]:Tianjin Tianjin. Tianjin University of Technology 2017

[2] Eleonora B, Laura M, Claudio C, et al. Beta rhythm modulation by speech sounds: somatotopic mapping in somatosensory cortex:[J]. Sci Rep, 2016, 6:31182.

[3] Juczewski K, Von R H, Bagni C, et al. Somatosensory map expansion and altered processing of tactile inputs in a mouse model of fragile $\mathrm{X}$ syndrome[J]. Neurobiology of Disease, 2016, 96:201-215.

[4] Research on the development steps of Android mobile phone application based on Xunfei Voice [J] Wireless Internet Technology,2015(14)123124

[5] Liu X, Ge S S, Jiang R, et al. Intelligent speech control system for human-robot interaction[C]. Control Conference. IEEE, 2016:61546159.

[6] Yao J, Liu Y, Shi Y. A Speech Control System for Intelligent Industry[C]. International Conference on Computer and Information Technology Applications. 2016.

[7] Deng Yang. Design and implementation of voice control system based on Android platform [D] Shenyang, Northeastern University, 2014

[8] Xue H, University S. The Application of iFLYTEK's Speech Technology in Mobile Phone APP for Meeting Minutes[J]. Computer \& Telecommunication, 2017.

[9] He Z, Lv P, Li W, et al. A synchronized pruning composition algorithm of weighted finite state transducers for large vocabulary speech recognition[C]. International Symposium on Chinese Spoken Language Processing. IEEE, 2013:11-15.

[10] Wang Shuai .Design of Remote Monitoring Mode for Greenhouse Environment Based on Android [J] 2017,9,27-29

[11] Sikarwar S, Yadav B C. Opto-electronic humidity sensor: A review[J]. Sensors \& Actuators A Physical, 2015, 233:54-70.

[12] Yu Yixiao, Zheng Qilin, Cheng Xinyu. Realization of smart home system based on speech recognition and MQTT protocol [J], 2017,11,97-99

[13] Singh M, Rajan M A, Shivraj V L, et al. Secure MQTT for Internet of Things (IoT)[C]. Fifth International Conference on Communication Systems and Network Technologies. IEEE, 2015:746-751.

[14] Sun Junlong. Mobile robot remote video transmission control research [D] Nanchang, Nanchang University 2016

[15] Frejlichowski D. Application of foreground object patterns analysis for event detection in an innovative video surveillance system[J]. Pattern Analysis \& Applications, 2015, 18(3):473-484. 